\title{
Quantitative analysis of some important metals and metalloids in tobacco products by inductively coupled plasma-mass spectrometry (ICP-MS)
}

Syed Ghulam Musharraf ${ }^{* *}$, Muhammad Shoaib², Amna Jabbar Siddiqui', Muhammad Najam-ul-Haq² and Aftab Ahmed ${ }^{3}$

\begin{abstract}
Background: Large scale usage of tobacco causes a lot of health troubles in human. Various formulations of tobacco are extensively used by the people particularly in developing world. Besides several toxic tobacco constituents some metals and metalloids are also believed to pose health risks. This paper describes inductively coupled plasma-mass spectrometric (ICP-MS) quantification of some important metals and metalloids in various brands of smoked, sniffed, dipped and chewed tobacco products.

Results: A microwave-assisted digestion method was used for sample preparation. The method was validated by analyzing a certified reference material. Percentage relative standard deviation (\% R.S.D.) between recovered and certified values was $<$ 5.8. Linearity value for calibration curve of each metal was $1>r>0.999$. Improved limits of detection (LODs) were in range of $\mathrm{ng} / \mathrm{L}$ for all elements. Fe, Al and $\mathrm{Mn}$ were found to be in the highest concentration in all types of tobacco products, while $\mathrm{Zn}, \mathrm{Cu}, \mathrm{Ni}$ and $\mathrm{Cr}$ were below the average concentration of $40 \mu \mathrm{g} / \mathrm{g}$, and $\mathrm{Pb}, \mathrm{Co}, \mathrm{As}$, Se and Cd were below $5 \mu \mathrm{g} / \mathrm{g}$. All elements, apart from Pb, were high in concentration in dipping tobacco in comparison to other tobacco products. Generally, the order of all elemental concentration can be expressed in different tobacco products as chewing $<$ smoked $<$ sniffing $<$ dipping. However, smoked and sniffing will interchange their position in the case of $\mathrm{Mn}, \mathrm{Cu}$, Se and $\mathrm{Cd}$. Multivariate statistical analyses were also performed to evaluate the correlation and variations among tobacco products.
\end{abstract}

Conclusions: The present study highlights the quantification of some important metals and metalloids in a wide spectrum of tobacco formulations. The outcome of this study would be beneficial for health authorities and individuals.

Keywords: Metals, Metalloids, Tobacco products, ICP-MS

\section{Background}

Tobacco use, especially in Asian countries has a long history. Five million deaths per annum, globally, are attributed to tobacco use. If this tendency continues, tobacco related mortalities will reach 8 million worldwide annually by the year 2030 [1]. Leaves of tobacco plants are used to prepare different products including smoked (cigarettes, beedi, tobacco leaves) and smokeless (sniffing, dipping, chewing) tobacco products. The composition of

\footnotetext{
* Correspondence: musharraf1977@yahoo.com

${ }^{1}$ H.E.J. Research Institute of Chemistry, International Center for Chemical and Biological Sciences, University of Karachi, Karachi 75270, Pakistan Full list of author information is available at the end of the article
}

tobacco is multifarious. The type and number of chemical constituents varies in its different formulations. Thousands of different chemicals have been detected in tobacco smoke; $60-70$ of them are proven carcinogens [2]. The only chemical which causes addiction in tobacco products is nicotine [3]; however the tobacco plant is well known to absorb trace elements from the soil and to accumulate them in its leaves on large scale. Some of these elements are toxic to human even in minute quantities [4-7]. Quantity of these trace elements in tobacco depends upon genotype, type of water, soil and their respective $\mathrm{pH}$, stalk position, fertilizers, pesticides applied and the environment.

\section{(Chemistry Central}


Biochemical effects of toxic and trace elements in tobacco and tobacco smoke are well documented by Chiba [8]. Among the metals, aluminum is the major ingredient in tobacco formulations. Aluminum toxicity is associated with alteration of calcium metabolism in the brain [9]. Chromium is carcinogenic in its hexavalent form. Maternal smoking has been linked to adverse effects on selenium metabolism in the developing foetus. Such women have low polymorphonuclear cell zinc concentrations, ultimately face a threat of delivering smallfor-gestational-age babies [8]. The International Agency for Research on Cancer (IARC) has recently defined nickel as a Group 1 "carcinogenic to humans" [10]. Copper poisoning in humans, particularly by chewing the tobacco formulation called gutka, is a major source of fibrosis in mouth cavities [11]. Lead is more hazardous for the young ones, as its chronic exposure plays role in the lowering of intelligence quotient (IQ) levels and it is also associated with impaired foetal development [12]. Arsenic toxicity causes skin cancer, mouth ulcerations, low haemoglobin, leukaemia, acute renal failure and nerve damages [13]. High cadmium level is related to cardiovascular diseases [14].

Pakistan produces more than 75 million kilograms of tobacco annually. After meeting the domestic requirement of 45-50 million kilograms, the rest of the tobacco is exported. Tobacco utilization is constantly increasing in Pakistan, India, Bangladesh and other countries of South Asia. In Pakistan, there are 22 million smokers, according to a health survey by Pakistan Medical Research Council in 2003, 36\% of adult men and 9\% of adult women are smokers in Pakistan [15] and about 100,000 people die in the country each year from the diseases caused by tobacco use. Tobacco formulations such as niswar and gutka are popular among the people of South Asia and responsible for oral cancer and its related diseases. Niswar, a sniffing and dipping tobacco product, is mainly composed of tobacco leaves, calcium oxide, and wood ash while Gutka, a chewing tobacco product, is composed of crushed areca nut, tobacco, catechu, paraffin, lime and sweet syrup. Gutka is very popular among teenagers, whilst niswar is particularly used in the Pushtoon and Afghan communities in Pakistan. These tobacco product usages are increasing at an alarming rate. Moreover sniffing, dipping and chewing products may cause metal toxicity as these products are directly consumed by the users and metals can absorb directly through the mucosal membrane. Therefore, there is an important need to quantify the metals, particularly in sniffing, dipping and chewing tobacco products.

Many methods have been employed for the determination of metals in tobacco products, mainly in cigarettes [16-25]. The aim of the present work was to develop a sensitive method for the quantitative estimation of a wide range of metals and two important metalloids (As \& Se) in a variety of tobacco products and to compare levels. Twelve elements, in a broad collection of Pakistani cigarette brands, niswar, sniffing niswar, and gutka formulations, were analyzed by ICP-MS using microwave digestion. International cigarettes and niswar formulations were also analyzed for a comparison of metal and metalloid's toxicity of local and imported tobacco products. Moreover, the present study will be helpful to pick dietary intake values of these metals and metalloids, so that one can assess the risk of overdosing of these metals and metalloids. It is the first report for the determination of metals and metalloids in a wide range of tobacco products and it will be useful for health and environment authorities in Pakistan and other countries of South Asia.

\section{Experimental}

\section{Chemicals and instrumentation}

Nitric acid was of trace metal grade and was purchased from Thermo-Fisher, USA. ICP-MS verification standard (Tune-A) and multi-element calibration standards were purchased from Ultra Scientific, USA. Deionized water from Milli-Q system (Millipore, Bedford, MA, USA) was used exclusively. Samples were digested using the Microwave Accelerated Reaction System and associated Teflon microwave vessels (CEM Corp, Matthews, USA, X7Series). Elemental analysis was performed using Thermo Elemental X7 Series ICP-MS System coupled with Cetac ASX-510HS high speed auto sampler (Omaha, Nebraska 68144 USA). Argon gas cylinders were connected through a six cylinder gas manifold from Western Innovator (Ohio, USA). Samples were centrifuged by Eppendorf micro-centrifuge (Hamburg, Germany).

\section{Sampling}

Fifty five different brands of tobacco smoked, sniffing, dipping and chewing products, commercially available in Pakistan, were purchased from various markets of Karachi. Each product was purchased twice, having different batch numbers and packing dates from different shops and stored in plastic bags separately. Envelope, paper and filters were carefully removed from the cigarettes and weighed amounts of duplicate samples were pooled and crushed in liquid nitrogen with pestle and mortar. Fine powdered samples were air-dried and stored at $4{ }^{\circ} \mathrm{C}$ in polythene bags until use. Sniffing, dipping and chewing samples were processed similarly and stored. All samples were coded and their decoding details are available in the Additional file 1.

\section{Microwave-assisted digestion}

Samples (10-30 mg) were weighted directly into microvessels and $1 \mathrm{~mL}$ of nitric acid was added. After five 
minutes, vials were capped using a torque wrench of capacity $20 \mathrm{in}-\mathrm{lb}(\cong 2.26 \mathrm{Nm})$ with a $22 \mathrm{~mm}$ socket. $10 \mathrm{~mL}$ of water was added into each HP500 plus standard digestion vessel. Two Teflon reaction vessels were inserted carefully in each digestion vessel. The microassisted digestion parameters were as follows: $\max$ power $600 \mathrm{~W}$, ramp time $10 \mathrm{~min}$, pressure $350 \mathrm{psi}$, and temperature $130{ }^{\circ} \mathrm{C}$ for $10 \mathrm{~min}$. Upon completion of the digestion process, vessels were allowed to cool at room temperature for $30 \mathrm{~min}$. Digested materials were transferred to Eppendorf tubes and centrifuged at 10,000 rpm for three minutes to remove any undigested material. Digested materials $(200 \mu \mathrm{L})$ were diluted in $9.8 \mathrm{~mL}$ of deionized water in $15 \mathrm{~mL}$ tubes and then analyzed by ICP-MS. To avoid contamination of samples, all PTFE material (Teflon vessels, pipettes, micro pipettes, tips and autosampler cups) were immersed in freshly prepared $15 \% \mathrm{v} / \mathrm{v} \mathrm{HNO}_{3}$ for $2 \mathrm{~h}$ before analysis, then rinsed thoroughly with deionized water and dried in a dust free area before use. The blank digests and filtered residues went through a similar protocol of sample preparation and all samples were blank-corrected. In the case of filtered residue extracts (filtering), the concentration of most elements were below detection limits and in exceptional cases very close to the detection limits, indicating that metallic contents staying behind in undigested material had negligible significance.

\section{ICP-MS analysis}

All samples, standards and reference materials, were analyzed in triplicate. An instrument auto-tune was performed using instrument verification standard (Tune-A) at $10 \mathrm{ppb}$ in $2 \%$ nitric acid. A multi-element six point calibration standard curve was generated at 50, 100, 150, 200, 250 and $300 \mathrm{ppb}$. Samples were diluted in $2 \%$ nitric acid and aspirated by using the auto-sampler. The concentration was calculated from the dilution corrected values of the elements. Complete instrumental parameters are highlighted in (Table 1). Statistical evaluation of the results was carried out by Microsoft office Excel 2003. The proposed method was validated by undertaking a comparative study of the microwaveassisted digestion against certified reference material; Oriental tobacco leaves (CTA-OTL-1). Certified reference material was microwave-assisted digested and further analyzed by ICP-MS. Percentage relative standard deviation (\% R.S.D.) was calculated by using recovered value of each metal from the proposed method and certified value. The percentage recovery of each metal was calculated as:

$$
\begin{gathered}
\% \text { Recovery }=100 \times \text { value of proposed method } \\
\text { /certified value }
\end{gathered}
$$

Table 1 Operating conditions for Plasma \& ICP-MS

\begin{tabular}{ll}
\hline Instrument & $\begin{array}{l}\text { X7-Series ICP-MS System, } \\
\text { Thermo Elemental Software, } \\
\text { Version 131072 }\end{array}$ \\
\hline Power & $1403.92 \mathrm{~W}$ \\
\hline Cool gas flow rate & $13 \mathrm{~L} / \mathrm{min}$ \\
\hline Nebuilizer gas pressure & $0.84 \mathrm{bar}$ \\
\hline Nebuilizer gas flow rate & $0.82 \mathrm{~L} / \mathrm{min}$ \\
\hline Auxiliary gas flow rate & $0.7 \mathrm{~L} / \mathrm{min}$ \\
\hline Condenser temperature & $15^{\circ} \mathrm{C}$ \\
\hline Extract lens 1 voltage & $3.8 \mathrm{~V}$ \\
\hline Extract lens 2 voltage & $-105.1 \mathrm{~V}$ \\
\hline Extract lens 3 voltage & $-195.29 \mathrm{~V}$ \\
\hline Pole bias & $1.53 \mathrm{~V}$ \\
\hline Hexapole bias & $6.47 \mathrm{~V}$ \\
\hline Data acquisition & Using peri pump at 31 rpm \\
\hline Main run setup & Peak jumping, Sweeps 100, \\
& $\begin{array}{l}\text { Dwell time 10 (ms), Channels } \\
\text { per mass 1, Channel spacing } \\
0.02 \mathrm{AMU}\end{array}$ \\
\hline No. of replicates & 3 \\
\hline Sampler cone i.d. & $1.1 \mathrm{~mm}$ \\
\hline Skimmer cone i.d. & $0.75 \mathrm{~mm}$ \\
\hline External drift correction & Yes \\
\hline
\end{tabular}

\section{Statistical analysis}

Multivariate statistical analysis can help to interpret data in a much easier and understandable manner. In the present study, simple XLSTAT (version 2011.4.04) was used for three multivariate techniques. There were 12 columns expressing elements and 55 rows indicating individual products. For a detailed statistical evaluation, principal component analysis was done on the whole data. It was done by diagonalizing the correlation matrix and all values were set at maximum unit variance, so the difficulty in analyzing different ranges of data points was avoided. The variability of the whole data was projected onto a scale, dividing variance into sub classes called principal components or factors. Moreover, Q-mode factor analysis after Varimax rotation was performed to check the variability among the tobacco products.

\section{Results and discussion}

\section{Analytical figures of merit}

The calibration curve for each metal and metalloid was constructed by using concentration range from the detection limit up to 50, 100, 150, 200, 250 and $300 \mathrm{ppb}$. The linearity, $(r)$ value in all cases was $1>r>0.999$. The limit of detection, LOD, was determined by aspiring ultra pure water as blank and signal intensities were recorded. A solution of $5 \mu \mathrm{g} / \mathrm{L}$ for each element was aspired and the signal intensities for all metals 
and metalloids were recorded. LOD was calculated by using equation:

$$
L O D=3 . S D_{\text {blank }} \cdot \text { Conc.sample } / I_{\text {net }}
$$

Where $\mathrm{SD}_{\text {blank }}$ is the standard deviation for the signal recorded on the blank for the corresponding element studied, Concn.sample is concentration in $\mu \mathrm{g} / \mathrm{L}$ of the respective sample aspired, $I_{\text {net }}=\left[\mathrm{I}_{\text {sample }}-\mathrm{I}_{\text {blank }}\right], \mathrm{I}_{\text {sample }} \&$ $\mathrm{I}_{\text {blank }}$ are the signal intensities recorded for the sample and blank, respectively. The LODs for all metals are summarized in (Table 2). Moreover, all samples above their LOD can be quantified, if their quantification limit would be approximately ten times the limit of detection.

\section{Recovery study}

Recoveries of target elements were computed by comparison of microwave-assisted digestion data against certified reference material (CRM) values. Results showed that there was a good agreement between the recovered and certified values of the metal and metalloid contents in certified reference material. Percentage recovery of all elements was $96.1<\mathrm{M}<108.5 \%$, while percentage relative standard deviation (\% R.S.D.) was $<5.8$ in all cases. $\%$ Recovery of metal and metalloid content as evaluated by comparing the recovered and certified values is summarized in (Table 3).

\section{Quantification of metals and metalloids in various tobacco products \\ Tobacco smoked products}

Thirty-five samples of tobacco smoked products, including 26 Pakistani, 6 foreign brands of cigarettes, 2 brands of beedi and tobacco leaves (used for hookah smoking) were investigated for the quantification of metals and

Table 2 Linear regression data for the calibration curve of each element $(n=3)$

\begin{tabular}{lccccc}
\hline Element & Linearity, $\mathbf{r}$ & SD blank $^{\mathbf{a}}$ & $\begin{array}{l}\mathbf{I}_{\text {blank }} \\
\text { (CPS) }^{\mathbf{b}}\end{array}$ & $\begin{array}{l}\mathbf{I}_{\text {net }} \\
(\mathbf{C P S})^{\mathbf{b}}\end{array}$ & $\begin{array}{l}\text { LOD } \\
\text { (ng/L) }\end{array}$ \\
\hline $\mathrm{Al}$ & 0.999997 & 1.388 & 420199.36 & 26159.85 & 0.796 \\
\hline $\mathrm{Cr}$ & 0.999969 & 0.023 & 8422.57 & 45971.71 & 0.008 \\
\hline $\mathrm{Mn}$ & 0.999493 & 0.049 & 16561.75 & 63241.03 & 0.012 \\
\hline $\mathrm{Fe}$ & 0.999989 & 0.417 & 602352.49 & 59517.32 & 0.100 \\
\hline $\mathrm{Co}$ & 0.999830 & 0.005 & 661.02 & 55386.24 & 0.001 \\
\hline $\mathrm{Ni}$ & 0.999907 & 0.038 & 4008.22 & 12804.31 & 0.045 \\
\hline $\mathrm{Cu}$ & 0.999901 & 0.023 & 2908.8 & 15433.35 & 0.022 \\
\hline $\mathrm{Zn}$ & 0.999973 & 0.058 & 844.04 & 9382.04 & 0.093 \\
\hline $\mathrm{As}$ & 0.999986 & 0.069 & -1311.36 & 8012.03 & 0.129 \\
\hline $\mathrm{Se}$ & 0.999905 & 0.255 & -42.93 & 730.7 & 5.235 \\
\hline $\mathrm{Cd}$ & 0.999980 & 0.008 & 58.33 & 8784.3 & 0.014 \\
\hline $\mathrm{Pb}$ & 0.999935 & 0.007 & 37.33 & 28190.14 & 0.004 \\
\hline${ }^{\mathrm{a} S t a n d a r d}$ deviation of twelve measurements; ${ }^{\mathrm{b}}$ Counts per second. &
\end{tabular}

Table 3 Validation of the micro-wave assisted digestion method against certified reference material (Oriental tobacco leaves, CTA-OTL-1), $n=6$

\begin{tabular}{lcclc}
\hline Element & $\begin{array}{l}\text { Certified } \\
\text { value } \pm \text { S.D. } \\
(\boldsymbol{\mu g} / \mathbf{g})\end{array}$ & $\begin{array}{l}\text { Recovered } \\
\text { value } \pm \text { S.D. } \\
(\boldsymbol{\mu g} / \mathbf{g})\end{array}$ & $\begin{array}{l}\text { \% } \\
\text { R.S.D. }\end{array}$ & $\begin{array}{l}\text { \% } \\
\text { Recovery }\end{array}$ \\
\hline${ }^{27} \mathrm{Al}$ & $252 \pm 49$ & $264.03 \pm 5.14$ & 3.29 & 104.77 \\
\hline${ }^{52} \mathrm{Cr}$ & $0.991^{\mathrm{a}}$ & $1.07 \pm 0.07$ & 5.42 & 107.97 \\
\hline${ }^{55} \mathrm{Mn}$ & $136 \pm 5$ & $141.59 \pm 3.2$ & 2.85 & 104.11 \\
\hline${ }^{56} \mathrm{Fe}$ & $258^{\mathrm{a}}$ & $248.11 \pm 4.77$ & 2.76 & 96.17 \\
\hline${ }^{59} \mathrm{Co}$ & $0.154^{\mathrm{b}} \pm 0.007$ & $0.167^{\mathrm{b}} \pm 0.009$ & 5.73 & 108.44 \\
\hline${ }^{60} \mathrm{Ni}$ & $1.49 \pm 0.14$ & $1.525 \pm 0.076$ & 1.64 & 102.35 \\
\hline${ }^{65} \mathrm{Cu}$ & $5.12 \pm 0.2$ & $5.48 \pm 0.02$ & 4.8 & 107.03 \\
\hline${ }^{66} \mathrm{Zn}$ & $43.6 \pm 1.4$ & $44.85 \pm 0.05$ & 1.99 & 102.87 \\
\hline${ }^{75} \mathrm{As}$ & $0.138^{\mathrm{b}} \pm 0.01$ & $0.144^{\mathrm{b}} \pm 0.01$ & 3 & 104.35 \\
\hline${ }^{82} \mathrm{Se}$ & $\mathrm{NA}^{\mathrm{c}}$ & $\mathrm{BDL}^{\mathrm{d}}$ & $\mathrm{NA}$ & $\mathrm{NA}$ \\
\hline${ }^{111} \mathrm{Cd}$ & $2.23 \pm 0.12$ & $2.386 \pm 0.005$ & 4.78 & 106.99 \\
\hline${ }^{208} \mathrm{~Pb}$ & $0.972^{\mathrm{b}} \pm 0.147$ & $0.965^{\mathrm{b}} \pm 0.028$ & 0.51 & 99.28 \\
\hline
\end{tabular}

${ }^{\mathrm{a}}$ Information value; ${ }^{\mathrm{b}} \mathrm{ng} / \mathrm{g} ;{ }^{\mathrm{c}}$ Not available; ${ }^{\mathrm{d}}$ Below detection limit.

metalloids by the developed method. Iron and aluminum were the major elements among the all analyzed elements and were found in the range of 190-2600 and $150-2100 \mu \mathrm{g} / \mathrm{g}$, respectively. Mn was in the range of $53-300 \mu \mathrm{g} / \mathrm{g}$ as the third most abundant element in all smoked tobacco products, while $\mathrm{Pb}, \mathrm{Cd}, \mathrm{Se}, \mathrm{Co}$, and $\mathrm{As}$ were present in $<3.4 \mu \mathrm{g} / \mathrm{g}$. Fe and $\mathrm{Al}$ having similar a range in national and foreign smoked brands, but their quantity was high in beedi samples. Foreign cigarette brands contain more $\mathrm{Mn}$ and $\mathrm{Zn}$ contents as compared to other smoked tobacco products. There was no significant difference in $\mathrm{Ni}$ contents of different types of smoked tobacco products. As and Cd have almost similar levels in different smoked tobacco products. Se was found in trace quantities in all tobacco smoked products and it was below the detection limit in some cases. $\mathrm{Pb}$ contents have similar trends in Pakistani cigarette brands, but in the case of foreign brands there were variations in its concentration. Metal and metalloid contents in all tobacco products are summarized in (Table 4) and graphically presented in Figure 1.

\section{Sniffing tobacco brands}

Three local samples of sniffing tobacco (sniffing niswar) brands were investigated for the quantification of metals and metalloids by the proposed method. $\mathrm{Fe}, \mathrm{Al}$ and $\mathrm{Mn}$ were the most abundant elements and present in higher concentrations in comparison to tobacco smoked products. Mn was found in the range of 79.2-83.6 $\mu \mathrm{g} / \mathrm{g}$, and lower in concentration in comparison to tobacco smoked brands. In the sniffing tobacco products, $\mathrm{Pb}$ was found in the range of 2.07-3.99 $\mu \mathrm{g} / \mathrm{g}$, more than that of smoked tobacco products. $\mathrm{Cd}$ was the minor trace 
Table 4 Metals and metalloids content in various tobacco products

\section{Code}

\begin{tabular}{|c|c|c|c|c|c|c|c|c|c|c|c|c|}
\hline \multirow[t]{2}{*}{ Code } & \multicolumn{12}{|c|}{ Amount of element $\mu \mathrm{g} / \mathrm{g} \pm$ S.D. } \\
\hline & Al & $\mathrm{Cr}$ & $\mathrm{Mn}$ & $\mathrm{Fe}$ & Co & $\mathrm{Ni}$ & $\mathrm{Cu}$ & $\mathrm{Zn}$ & As & Se & $\mathrm{Cd}$ & $\mathrm{Pb}$ \\
\hline \multicolumn{13}{|c|}{ Smoked tobacco products } \\
\hline $\mathrm{T1}$ & $820 \pm 5.00$ & $3.10 \pm 0.02$ & $110 \pm 0.90$ & $1200 \pm 8.46$ & $0.83 \pm 0.01$ & $3.92 \pm 0.10$ & $24.5 \pm 0.11$ & $38.6 \pm 0.15$ & $0.65 \pm 0.02$ & $\mathrm{BDL}$ & $0.68 \pm 0.01$ & $1.42 \pm 0.01$ \\
\hline $\mathrm{T} 2$ & $360 \pm 0.50$ & $1.49 \pm 0.05$ & $145 \pm 0.30$ & $460 \pm 0.41$ & $0.51 \pm 0.02$ & $2.36 \pm 0.06$ & $23.0 \pm 0.06$ & $34.0 \pm 0.20$ & $0.40 \pm 0.02$ & $0.29 \pm 0.01$ & $0.80 \pm 0.04$ & $0.65 \pm 0.01$ \\
\hline $\mathrm{T3}$ & $1000 \pm 1.47$ & $3.53 \pm 0.05$ & $120 \pm 0.15$ & $1500 \pm 0.75$ & $1.00 \pm 0.01$ & $3.94 \pm 0.11$ & $34.0 \pm 0.20$ & $45.0 \pm 0.25$ & $0.77 \pm 0.12$ & $\mathrm{BDL}$ & $0.61 \pm 0.02$ & $1.89 \pm 0.01$ \\
\hline $\mathrm{T4}$ & $603 \pm 1.33$ & $3.21 \pm 0.06$ & $90.4 \pm 0.10$ & $905 \pm 3.55$ & $0.64 \pm 0.03$ & $4.19 \pm 0.20$ & $18.8 \pm 0.20$ & $26.5 \pm 0.23$ & $0.91 \pm 0.09$ & $1.25 \pm 0.06$ & $0.54 \pm 0.03$ & $1.09 \pm 0.05$ \\
\hline T5 & $635 \pm 14.30$ & $2.76 \pm 0.05$ & $199.6 \pm 0.4$ & $872 \pm 18.20$ & $0.78 \pm 0.01$ & $3.94 \pm 0.26$ & $22.4 \pm 0.36$ & $34.4 \pm 1.19$ & $0.63 \pm 0.02$ & $\mathrm{BDL}$ & $1.10 \pm 0.05$ & $1.59 \pm 0.05$ \\
\hline T6 & $870 \pm 5.50$ & $3.52 \pm 0.02$ & $131.1 \pm 0.3$ & $1300 \pm 5.70$ & $0.90 \pm 0.01$ & $4.26 \pm 0.05$ & $23.8 \pm 0.15$ & $33.9 \pm 0.10$ & $0.87 \pm 0.04$ & $0.64 \pm 0.21$ & $0.66 \pm 0.03$ & $1.29 \pm 0.01$ \\
\hline $\mathrm{T7}$ & $588 \pm 2.78$ & $2.45 \pm 0.05$ & $170 \pm 0.92$ & $790 \pm 1.21$ & $0.68 \pm 0.01$ & $2.24 \pm 0.15$ & $16.43 \pm 0.1$ & $35.4 \pm 0.17$ & $0.39 \pm 0.05$ & BDL & $1.00 \pm 0.02$ & $0.92 \pm 0.01$ \\
\hline$\overline{T 8}$ & $1150 \pm 16.00$ & $3.44 \pm 0.05$ & $105 \pm 1.47$ & $1400 \pm 17.7$ & $0.81 \pm 0.01$ & $3.61 \pm 0.07$ & $24.2 \pm 0.30$ & $36.7 \pm 3.21$ & $0.87 \pm 0.07$ & $0.66 \pm 0.14$ & $0.52 \pm 0.01$ & $1.18 \pm 0.05$ \\
\hline T9 & $517 \pm 6.39$ & $1.92 \pm 0.09$ & $142 \pm 1.59$ & $724 \pm 5.09$ & $0.64 \pm 0.02$ & $2.33 \pm 0.20$ & $15.75 \pm 0.1$ & $32.9 \pm 0.10$ & $0.89 \pm 0.11$ & $1.60 \pm 0.14$ & $1.22 \pm 0.10$ & $0.78 \pm 0.01$ \\
\hline $\mathrm{T} 10$ & $803 \pm 8.82$ & $3.17 \pm 0.05$ & $103 \pm 0.75$ & $1200 \pm 9.53$ & $0.85 \pm 0.01$ & $4.15 \pm 0.15$ & $26.9 \pm 0.15$ & $38.4 \pm 0.43$ & $0.81 \pm 0.09$ & $0.24 \pm 0.09$ & $0.63 \pm 0.05$ & $1.37 \pm 0.05$ \\
\hline $\mathrm{T} 11$ & $507 \pm 15.60$ & $3.07 \pm 0.05$ & $76.8 \pm 0.26$ & $840 \pm 1.20$ & $0.58 \pm 0.01$ & $2.33 \pm 0.10$ & $21.9 \pm 0.05$ & $29.5 \pm 0.05$ & $0.57 \pm 0.09$ & $0.17 \pm 0.18$ & $0.36 \pm 0.03$ & $1.01 \pm 0.01$ \\
\hline $\mathrm{T} 12$ & $894.8 \pm 30.40$ & $2.05 \pm 0.14$ & $66.12 \pm 1.9$ & $886.8 \pm 28$ & $0.44 \pm 0.02$ & $2.18 \pm 0.21$ & $20.7 \pm 0.75$ & $32.44 \pm 1.4$ & $1.08 \pm 0.21$ & $0.50 \pm 0.12$ & $0.3 \pm 0.025$ & $0.87 \pm 0.05$ \\
\hline$\overline{\mathrm{T} 13}$ & $488 \pm 3.70$ & $3.00 \pm 0.05$ & $232 \pm 0.20$ & $623 \pm 1.34$ & $0.84 \pm 0.02$ & $2.52 \pm 0.10$ & $21.5 \pm 0.05$ & $52.5 \pm 0.20$ & $0.65 \pm 0.12$ & $0.62 \pm 0.15$ & $1.23 \pm 0.03$ & $0.61 \pm 0.01$ \\
\hline T14 & $520 \pm 4.51$ & $3.06 \pm 0.10$ & $78.9 \pm 0.66$ & $832 \pm 8.17$ & $0.60 \pm 0.01$ & $2.27 \pm 0.15$ & $16.5 \pm 0.15$ & $24.5 \pm 0.15$ & $0.51 \pm 0.07$ & $0.062 \pm 0.1$ & $0.43 \pm 0.01$ & $0.98 \pm 0.02$ \\
\hline T15 & $399 \pm 0.60$ & $2.08 \pm 0.10$ & $167 \pm 0.10$ & $541 \pm 1.73$ & $0.54 \pm 0.01$ & $2.27 \pm 0.17$ & $15.2 \pm 0.11$ & $35.8 \pm 0.25$ & $0.61 \pm 0.10$ & $0.73 \pm 0.07$ & $1.76 \pm 0.03$ & $1.08 \pm 0.10$ \\
\hline T16 & $1310 \pm 12.20$ & $4.25 \pm 0.05$ & $143 \pm 1.65$ & $1870 \pm 21.8$ & $1.30 \pm 0.02$ & $4.67 \pm 0.05$ & $21.9 \pm 0.20$ & $37.9 \pm 0.25$ & $1.00 \pm 0.10$ & $\mathrm{BDL}$ & $0.54 \pm 0.03$ & $1.72 \pm 0.01$ \\
\hline $\mathrm{T} 17$ & $1420 \pm 5.60$ & $4.19 \pm 0.05$ & $122 \pm 0.45$ & $1480 \pm 7.70$ & $0.85 \pm 0.01$ & $3.30 \pm 0.10$ & $22.0 \pm 0.10$ & $37.5 \pm 0.10$ & $0.81 \pm 0.07$ & $0.21 \pm 0.04$ & $0.47 \pm 0.02$ & $1.29 \pm 0.02$ \\
\hline $\mathrm{T} 18$ & $886 \pm 9.12$ & $2.93 \pm 0.02$ & $109.7 \pm 1.0$ & $935 \pm 10.80$ & $0.68 \pm 0.01$ & $3.06 \pm 0.10$ & $20.5 \pm 0.06$ & $29.8 \pm 0.10$ & $0.50 \pm 0.01$ & $\mathrm{BDL}$ & $0.73 \pm 0.03$ & $1.22 \pm 0.01$ \\
\hline T19 & $640 \pm 1.00$ & $3.50 \pm 0.10$ & $104 \pm 0.45$ & $1030 \pm 2.12$ & $0.74 \pm 0.02$ & $2.61 \pm 0.26$ & $19.0 \pm 0.17$ & $29.7 \pm 0.55$ & $0.86 \pm 0.03$ & $0.47 \pm 0.04$ & $0.45 \pm 0.04$ & $1.06 \pm 0.05$ \\
\hline $\mathrm{T} 20$ & $566 \pm 1.90$ & $2.93 \pm 0.05$ & $89.3 \pm 0.15$ & $844 \pm 2.02$ & $0.63 \pm 0.01$ & $2.25 \pm 0.23$ & $19.2 \pm 0.20$ & $27.0 \pm 0.23$ & $0.51 \pm 0.04$ & $\mathrm{BDL}$ & $0.50 \pm 0.03$ & $0.99 \pm 0.01$ \\
\hline T21 & $865 \pm 7.75$ & $3.67 \pm 0.05$ & $103 \pm 1.21$ & $1300 \pm 12.5$ & $0.84 \pm 0.01$ & $2.69 \pm 0.06$ & $19.2 \pm 0.10$ & $30.2 \pm 0.25$ & $0.94 \pm 0.10$ & $1.06 \pm 0.21$ & $0.55 \pm 0.01$ & $1.29 \pm 0.01$ \\
\hline T22 & $642 \pm 5.43$ & $2.79 \pm 0.06$ & $93.0 \pm 0.72$ & $887 \pm 8.63$ & $0.72 \pm 0.01$ & $2.72 \pm 0.10$ & $20.3 \pm 0.10$ & $27.0 \pm 0.26$ & $0.69 \pm 0.12$ & $0.64 \pm 0.07$ & $0.49 \pm 0.03$ & $1.00 \pm 0.01$ \\
\hline $\mathrm{T} 23$ & $449 \pm 7.09$ & $2.17 \pm 0.05$ & $118 \pm 2.04$ & $647 \pm 10.03$ & $0.70 \pm 0.01$ & $2.40 \pm 0.05$ & $20.4 \pm 0.30$ & $32.0 \pm 0.43$ & $0.46 \pm 0.06$ & $0.27 \pm 0.05$ & $0.74 \pm 0.03$ & $1.18 \pm 0.05$ \\
\hline $\mathrm{T} 24$ & $633 \pm 6.59$ & $2.90 \pm 0.06$ & $145 \pm 1.02$ & $967 \pm 7.55$ & $0.81 \pm 0.01$ & $3.66 \pm 0.06$ & $19.9 \pm 0.15$ & $35.3 \pm 0.10$ & $0.96 \pm 0.10$ & $1.10 \pm 0.07$ & $0.74 \pm 0.03$ & $1.19 \pm 0.10$ \\
\hline T25 & $669 \pm 3.41$ & $2.62 \pm 0.05$ & $91.96 \pm 0.4$ & $907 \pm 0.85$ & $0.68 \pm 0.01$ & $2.39 \pm 0.10$ & $19.0 \pm 0.15$ & $27.0 \pm 0.20$ & $0.60 \pm 0.07$ & $\mathrm{BDL}$ & $0.44 \pm 0.01$ & $1.06 \pm 0.03$ \\
\hline $\mathrm{T} 26$ & $382 \pm 0.62$ & $2.19 \pm 0.10$ & $170 \pm 0.10$ & $570 \pm 1.96$ & $0.86 \pm 0.01$ & $5.09 \pm 0.09$ & $21.5 \pm 0.37$ & $47.0 \pm 0.70$ & $0.60 \pm 0.03$ & $0.34 \pm 0.04$ & $1.27 \pm 0.04$ & $0.57 \pm 0.01$ \\
\hline 11 & $710 \pm 2.52$ & $1.84 \pm 0.07$ & $223 \pm 1.06$ & $600 \pm 1.05$ & $0.93 \pm 0.01$ & $2.23 \pm 0.05$ & $14.9 \pm 0.11$ & $53.3 \pm 0.23$ & $0.33 \pm 0.01$ & $\mathrm{BDL}$ & $0.96 \pm 0.03$ & $0.64 \pm 0.01$ \\
\hline 12 & $150 \pm 1.10$ & $1.68 \pm 0.05$ & $260 \pm 2.53$ & $190 \pm 1.36$ & $0.65 \pm 0.01$ & $1.92 \pm 0.07$ & $10.5 \pm 0.11$ & $52.6 \pm 0.20$ & $1.16 \pm 0.28$ & $2.28 \pm 0.28$ & $1.49 \pm 0.07$ & $1.03 \pm 0.02$ \\
\hline 13 & $320 \pm 1.94$ & $2.36 \pm 0.05$ & $225 \pm 1.93$ & $460 \pm 2.08$ & $0.89 \pm 0.01$ & $3.76 \pm 0.15$ & $17.4 \pm 0.11$ & $50.4 \pm 0.05$ & $0.82 \pm 0.13$ & $0.99 \pm 0.02$ & $3.35 \pm 0.05$ & $2.52 \pm 0.01$ \\
\hline 14 & $620 \pm 1.57$ & $1.66 \pm 0.05$ & $270 \pm 8.67$ & $570 \pm 3.55$ & $0.90 \pm 0.02$ & $2.43 \pm 0.05$ & $16.0 \pm 0.11$ & $42.2 \pm 0.15$ & $0.72 \pm 0.06$ & $0.39 \pm 0.02$ & $2.70 \pm 0.01$ & $2.36 \pm 0.05$ \\
\hline 15 & $300 \pm 3.81$ & $2.03 \pm 0.05$ & $200 \pm 2.90$ & $530 \pm 6.35$ & $0.76 \pm 0.01$ & $8.07 \pm 0.05$ & $15.0 \pm 0.30$ & $32.2 \pm 0.32$ & $0.38 \pm 0.02$ & $\mathrm{BDL}$ & $1.46 \pm 0.06$ & $0.56 \pm 0.01$ \\
\hline 16 & $330 \pm 4.73$ & $1.55 \pm 0.05$ & $300 \pm 13.60$ & $390 \pm 3.15$ & $1.03 \pm 0.01$ & $2.58 \pm 0.10$ & $17.0 \pm 0.15$ & $55.6 \pm 0.40$ & $0.72 \pm 0.27$ & $1.10 \pm 0.10$ & $3.20 \pm 0.05$ & $1.55 \pm 0.05$ \\
\hline
\end{tabular}


Table 4 Metals and metalloids content in various tobacco products (Continued)

\begin{tabular}{|c|c|c|c|c|c|c|c|c|c|c|c|c|}
\hline B1 & $360 \pm 3.50$ & $1.70 \pm 0.03$ & $53 \pm 0.25$ & $440 \pm 3.36$ & $0.52 \pm 0.02$ & $3.70 \pm 0.08$ & $11.0 \pm 0.24$ & $38.0 \pm 0.11$ & $1.30 \pm 0.28$ & $0.73 \pm 0.18$ & $0.27 \pm 0.03$ & $0.79 \pm 0.01$ \\
\hline B2 & $1900 \pm 30.20$ & $3.90 \pm 0.10$ & $89.9 \pm 1.19$ & $1600 \pm 20.0$ & $0.88 \pm 0.03$ & $3.60 \pm 0.07$ & $13.0 \pm 0.05$ & $31.0 \pm 0.40$ & $0.74 \pm 0.09$ & $0.89 \pm 0.13$ & $0.37 \pm 0.01$ & $1.32 \pm 0.03$ \\
\hline B3 & $2100 \pm 5.66$ & $5.37 \pm 0.10$ & $140 \pm 0.34$ & $2600 \pm 7.69$ & $1.31 \pm 0.02$ & $4.47 \pm 0.10$ & $13.7 \pm 0.14$ & $45.7 \pm 0.72$ & $1.53 \pm 0.18$ & $1.16 \pm 0.09$ & $0.50 \pm 0.08$ & $2.00 \pm 0.01$ \\
\hline \multicolumn{13}{|c|}{ Sniffing tobacco products } \\
\hline N1 & $1496 \pm 9.28$ & $6.33 \pm 0.05$ & $83.6 \pm 0.10$ & $1940 \pm 14.2$ & $0.98 \pm 0.01$ & $4.87 \pm 0.13$ & $12.8 \pm 0.05$ & $34.9 \pm 0.20$ & $0.87 \pm 0.10$ & $0.52 \pm 0.09$ & $0.42 \pm 0.02$ & $2.38 \pm 0.01$ \\
\hline N2 & $2080 \pm 15.00$ & $6.86 \pm 0.04$ & $82.3 \pm 0.51$ & $2220 \pm 8.57$ & $0.93 \pm 0.01$ & $5.07 \pm 0.09$ & $16.6 \pm 0.05$ & $40.8 \pm 0.15$ & $0.90 \pm 0.07$ & $0.59 \pm 0.01$ & $0.46 \pm 0.01$ & $2.07 \pm 0.02$ \\
\hline N3 & $1500 \pm 19.67$ & $7.14 \pm 0.13$ & $79.2 \pm 0.73$ & $1980 \pm 19.4$ & $0.93 \pm 0.01$ & $6.32 \pm 0.30$ & $13.6 \pm 0.23$ & $34.9 \pm 0.51$ & $0.77 \pm 0.18$ & $\mathrm{BDL}$ & $0.42 \pm 0.01$ & $3.99 \pm 0.05$ \\
\hline \multicolumn{13}{|c|}{ Dipping tobacco products } \\
\hline N4 & $1000 \pm 4.81$ & $2.89 \pm 0.03$ & $41.5 \pm 0.15$ & $908 \pm 5.36$ & $0.76 \pm 0.03$ & $2.52 \pm 0.15$ & $17.7 \pm 0.15$ & $17.3 \pm 0.30$ & $0.40 \pm 0.17$ & $0.37 \pm 0.12$ & $0.10 \pm 0.02$ & $0.53 \pm 0.01$ \\
\hline N5 & $670 \pm 1.22$ & $2.12 \pm 0.01$ & $36.6 \pm 0.11$ & $840 \pm 1.36$ & $0.41 \pm 0.01$ & $1.19 \pm 0.05$ & $37.9 \pm 0.15$ & $29.5 \pm 0.05$ & $0.58 \pm 0.17$ & $0.56 \pm 0.03$ & $0.19 \pm 0.01$ & $0.74 \pm 0.01$ \\
\hline N6 & $1400 \pm 15.50$ & $5.42 \pm 0.05$ & $42.8 \pm 0.32$ & $1440 \pm 12.2$ & $1.10 \pm 0.07$ & $3.60 \pm 0.02$ & $16.2 \pm 0.35$ & $15.4 \pm 0.05$ & $0.53 \pm 0.19$ & $0.38 \pm 0.02$ & $0.11 \pm 0.01$ & $0.69 \pm 0.02$ \\
\hline N7 & $6500 \pm 40.07$ & $12.8 \pm 0.10$ & $217 \pm 1.03$ & $7400 \pm 21.3$ & $2.47 \pm 0.02$ & $11.7 \pm 0.05$ & $31.9 \pm 0.50$ & $58.3 \pm 0.25$ & $2.39 \pm 0.15$ & $0.95 \pm 0.21$ & $0.25 \pm 0.01$ & $4.25 \pm 0.05$ \\
\hline N8 & $6400 \pm 6.87$ & $13.4 \pm 0.10$ & $183 \pm 0.35$ & $7340 \pm 23.6$ & $2.70 \pm 0.05$ & $10.5 \pm 0.05$ & $30.4 \pm 0.20$ & $63.2 \pm 0.10$ & $3.07 \pm 0.22$ & $1.66 \pm 0.35$ & $0.26 \pm 0.02$ & $4.90 \pm 0.25$ \\
\hline N9 & $4300 \pm 13.34$ & $52.0 \pm 0.28$ & $130 \pm 0.69$ & $4600 \pm 31.0$ & $2.17 \pm 0.01$ & $14.24 \pm 0.1$ & $13.8 \pm 0.15$ & $42.7 \pm 0.05$ & $1.39 \pm 0.10$ & $1.04 \pm 0.15$ & $0.15 \pm 0.01$ & $1.87 \pm 0.02$ \\
\hline N10 & $4500 \pm 15.89$ & $17.6 \pm 0.15$ & $178 \pm 0.90$ & $2130 \pm 13.7$ & $2.39 \pm 0.05$ & $9.98 \pm 0.20$ & $20.5 \pm 0.20$ & $49.8 \pm 0.10$ & $2.08 \pm 0.05$ & $1.67 \pm 0.35$ & $0.22 \pm 0.03$ & $4.27 \pm 0.05$ \\
\hline N11 & $3200 \pm 15.10$ & $8.17 \pm 0.06$ & $104 \pm 0.20$ & $3800 \pm 11.5$ & $1.62 \pm 0.01$ & $6.41 \pm 0.10$ & $14.9 \pm 0.10$ & $32.4 \pm 0.25$ & $1.74 \pm 0.17$ & $0.52 \pm 0.04$ & $0.14 \pm 0.01$ & $4.06 \pm 0.05$ \\
\hline $\mathrm{N} 12$ & $2420 \pm 16.20$ & $8.53 \pm 0.01$ & $77.5 \pm 0.35$ & $2440 \pm 10.9$ & $1.27 \pm 0.02$ & $6.09 \pm 0.13$ & $11.3 \pm 0.05$ & $23.4 \pm 0.11$ & $1.32 \pm 0.12$ & $1.31 \pm 0.01$ & $2.00 \pm 0.01$ & $2.44 \pm 0.02$ \\
\hline $\mathrm{N} 13$ & $5600 \pm 30.05$ & $78.8 \pm 0.15$ & $189.8 \pm 1.2$ & $6900 \pm 56.3$ & $2.90 \pm 0.07$ & $23.5 \pm 0.26$ & $20.9 \pm 0.15$ & $48.4 \pm 0.25$ & $1.89 \pm 0.20$ & $0.37 \pm 0.02$ & $0.21 \pm 0.02$ & $2.29 \pm 0.01$ \\
\hline IN1 & $2340 \pm 23.20$ & $11.4 \pm 0.05$ & $128.5 \pm 1.0$ & $2930 \pm 22.1$ & $1.30 \pm 0.01$ & $7.36 \pm 0.06$ & $12.6 \pm 0.03$ & $45.3 \pm 0.43$ & $1.33 \pm 0.08$ & $0.67 \pm 0.24$ & $0.65 \pm 0.04$ & $2.54 \pm 0.04$ \\
\hline IN2 & $1300 \pm 12.80$ & $4.80 \pm 0.06$ & $98.5 \pm 0.75$ & $1500 \pm 11.8$ & $0.74 \pm 0.01$ & $4.32 \pm 0.24$ & $12.2 \pm 0.05$ & $66.9 \pm 0.32$ & $1.08 \pm 0.20$ & $0.77 \pm 0.43$ & $0.61 \pm 0.02$ & $1.80 \pm 0.03$ \\
\hline IN3 & $2100 \pm 16.14$ & $9.06 \pm 0.47$ & $124 \pm 0.70$ & $2900 \pm 19.1$ & $1.25 \pm 0.01$ & $5.37 \pm 0.05$ & $13.1 \pm 0.15$ & $40.5 \pm 0.30$ & $1.81 \pm 0.17$ & $0.57 \pm 0.03$ & $0.62 \pm 0.02$ & $2.89 \pm 0.05$ \\
\hline \multicolumn{13}{|c|}{ Chewing tobacco products } \\
\hline G1 & $111.27 \pm 8.17$ & $0.69 \pm 0.07$ & $27.37 \pm 0.7$ & $176.96 \pm 5$ & $\mathrm{BDL}$ & $1.50 \pm 0.07$ & $16.0 \pm 0.55$ & $9.15 \pm 0.27$ & $\mathrm{BDL}$ & $1.03 \pm 0.11$ & $\mathrm{BDL}$ & $0.08 \pm 0.00$ \\
\hline G2 & $736.6 \pm 24.90$ & $10.44 \pm 0.3$ & $40.0 \pm 1.03$ & $762.1 \pm 20$ & $0.45 \pm 0.02$ & $9.27 \pm 0.51$ & $17.5 \pm 0.42$ & $17.07 \pm 0.6$ & $\mathrm{BDL}$ & $\mathrm{BDL}$ & $\mathrm{BDL}$ & $0.18 \pm 0.01$ \\
\hline G3 & $454 \pm 14.70$ & $1.95 \pm 0.10$ & $41.87 \pm 0.9$ & $563.1 \pm 13$ & $0.19 \pm 0.00$ & $2.75 \pm 0.17$ & $16.0 \pm 0.29$ & $47.72 \pm 1.1$ & $\mathrm{BDL}$ & $0.21 \pm 0.04$ & $\mathrm{BDL}$ & $1.85 \pm 0.07$ \\
\hline G4 & $542.7 \pm 20.70$ & $1.12 \pm 0.09$ & $51.07 \pm 1.3$ & $580.2 \pm 14$ & $0.06 \pm 0.01$ & $1.46 \pm 0.15$ & $14.68 \pm 0.4$ & $35.75 \pm 1.1$ & $\mathrm{BDL}$ & $0.07 \pm 0.01$ & $\mathrm{BDL}$ & $3.96 \pm 0.16$ \\
\hline
\end{tabular}



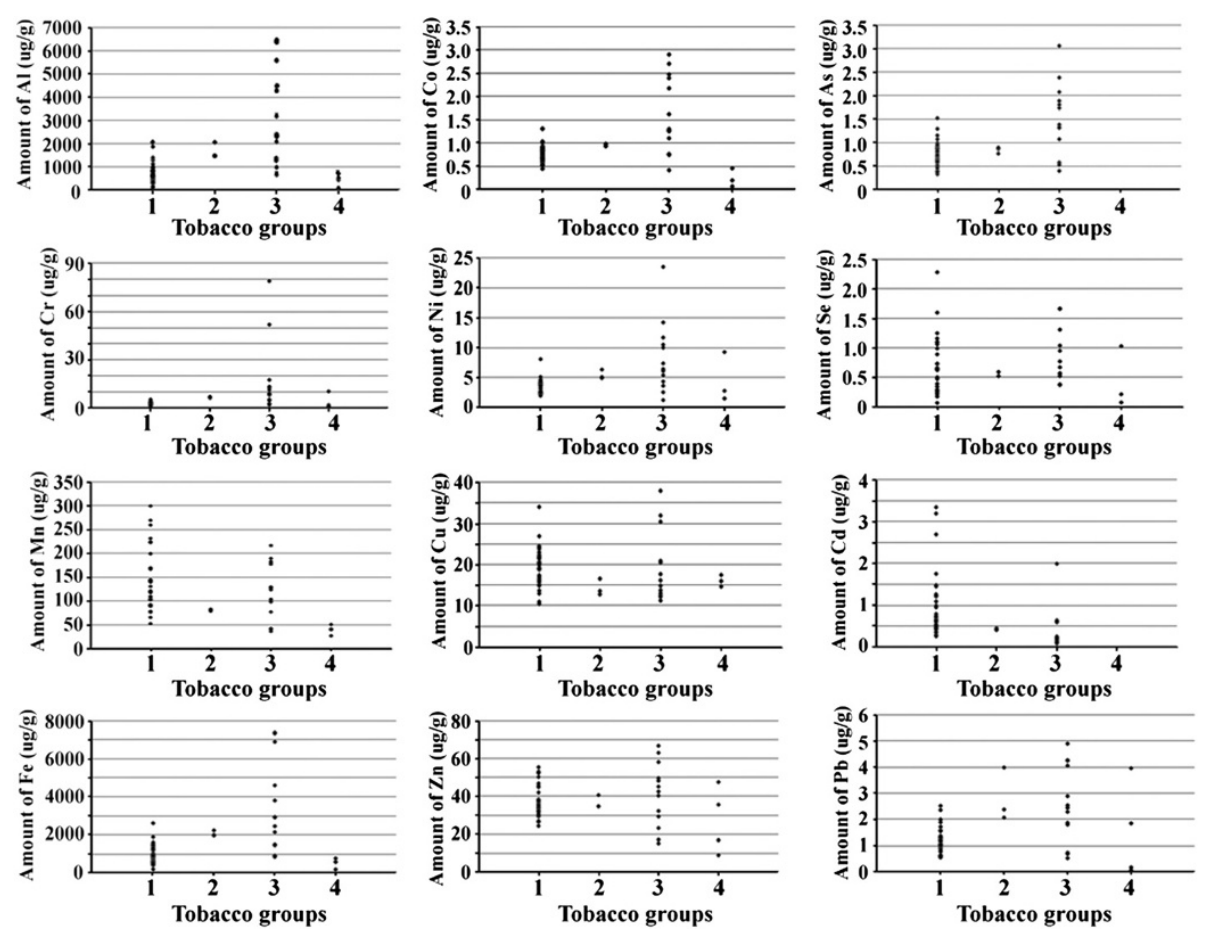

Figure 1 Graphical presentation of metals and metalloids contents in smoked 1, sniffing 2, dipping 3, and chewing 4 tobacco products.

metal, while Se was below the detection limit in sample N3. The results are presented in Table 4 and Figure 1.

\section{Dipping tobacco brands}

Ten local and three foreign samples of dipping tobacco (Niswar) brands were analyzed. $\mathrm{Fe}, \mathrm{Al}$ and $\mathrm{Mn}$ were the principal components also in dipping tobacco samples. $\mathrm{Fe}$ and $\mathrm{Al}$ were found in the ranges of $840-7400$ and $670-6500 \mu \mathrm{g} / \mathrm{g}$, respectively. Most of the dipping tobacco samples contained higher concentration of metal and metalloids than all other types of tobacco products, except Mn, Se and Cd. No significant difference of elemental contents was observed between local and imported dipping tobacco products except in two Pakistani products, N7 and N8 (Table 4) and Figure 1.

\section{Chewing tobacco brands}

Four brands of gutka (a chewing tobacco product), which is commonly consumed in Pakistan, were investigated. Generally, they contained metals in fewer quantities, except $\mathrm{Cr}, \mathrm{Ni}, \mathrm{Cu}, \mathrm{Zn}$ and Se. Three major elements $\mathrm{Fe}, \mathrm{Al}$ and $\mathrm{Mn}$, showed similar trends, to that

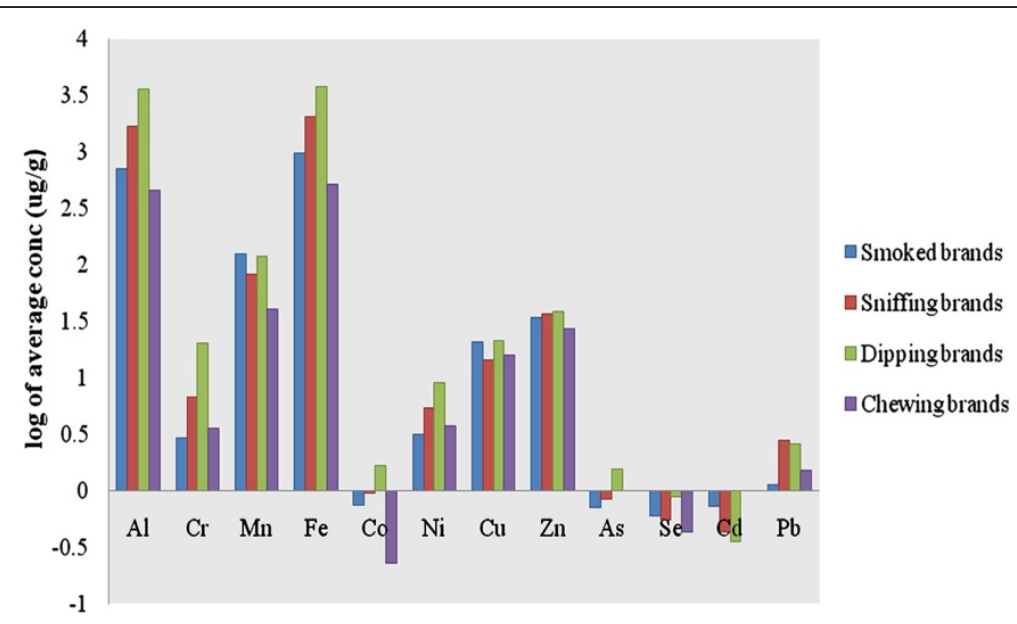

Figure 2 Graphical representation of average concentration of metals and metalloids in various tobacco groups. 
Table 5 Metal and metalloid concentration levels $(\mu \mathrm{g} / \mathrm{g})$ in reported literature

\begin{tabular}{|c|c|c|c|c|c|c|c|c|c|c|c|c|c|}
\hline Product & Al & $\mathrm{Cr}$ & $\mathrm{Mn}$ & $\mathrm{Fe}$ & Co & $\mathrm{Ni}$ & $\mathrm{Cu}$ & $\mathrm{Zn}$ & As & $\mathrm{Se}$ & $\mathrm{Cd}$ & $\mathrm{Pb}$ & Reference \\
\hline \multirow[t]{2}{*}{ Smoked } & - & - & 45.03 & - & 3.344 & - & 7.889 & 14.34 & - & - & 0.501 & 14.39 & 21 \\
\hline & 716 & 2.92 & 124.04 & 981.18 & 0.75 & 3.13 & 21.1 & 34.27 & 0.71 & 0.6 & 0.72 & 1.13 & Present study \\
\hline \multirow[t]{5}{*}{ Sniffing } & - & 15.6 & - & 2972 & - & 9.13 & 16 & 37 & - & - & 0.48 & 3.1 & 21 \\
\hline & - & $\sim 3.8$ & $\sim 124.4$ & - & $\sim 0.47$ & $\sim 3.0$ & $\sim 28$ & $\sim 42$ & - & - & - & $\sim 0.78$ & 30 \\
\hline & - & - & - & 2090 & - & 2.1 & 21 & 43 & - & - & - & 8.0 & 31 \\
\hline & - & 3.0 & 150 & 2323 & 0.4 & - & - & 46 & - & - & - & - & 32 \\
\hline & 1692 & 6.78 & 81.7 & 2046.67 & 0.95 & 5.42 & 14.33 & 36.87 & 0.85 & 0.56 & 0.43 & 2.81 & Present study \\
\hline Dipping & 3599 & 20.17 & 120.02 & 3779.8 & 1.68 & 8.97 & 21.55 & 38.04 & 1.54 & 0.89 & 0.36 & 2.6 & Present study \\
\hline \multirow[t]{6}{*}{ Chewing } & - & 6.97 & - & 853 & - & 2.37 & 42 & 56 & - & - & 0.38 & 8.38 & 29 \\
\hline & - & $\sim 6.0$ & $\sim 174$ & $\sim 1100$ & $\sim 0.69$ & $\sim 1.5$ & $\sim 12$ & $\sim 28$ & $\sim 0.91$ & - & $\sim 0.8$ & $\sim 4.5$ & 30 \\
\hline & - & - & - & 1050 & - & 1.1 & 11 & 20 & - & - & - & 4.5 & 31 \\
\hline & - & 6.3 & 127 & 1565 & 0.6 & - & - & 19 & - & - & - & - & 32 \\
\hline & - & 6.6 & 129 & 1703 & 0.75 & - & - & 18.5 & - & - & - & - & 33 \\
\hline & 461.14 & 3.55 & 40.08 & 520.59 & 0.23 & 3.75 & 16.05 & 27.42 & $\mathrm{BDL}$ & 0.44 & $\mathrm{BDL}$ & 1.52 & Present study \\
\hline
\end{tabular}

$B D L$ below detection limit.

observed in other tobacco products. A gutka sample, G2 had $\mathrm{Cr}$ and $\mathrm{Ni}$ in concentrations of 10.44 and $9.27 \mu \mathrm{g} / \mathrm{g}$, respectively, more than smoked and sniffing tobacco brands. Similarly, the amount of $\mathrm{Cu}(17.5 \mu \mathrm{g} / \mathrm{g})$ in $\mathrm{G} 2$ was higher than the sniffing tobacco products. The amount of $\mathrm{Zn}(47.72 \mu \mathrm{g} / \mathrm{g})$ in G3 was more than in the sniffing tobacco brands. Se $(1.03 \mu \mathrm{g} / \mathrm{g})$ in $\mathrm{G} 1$ was more than the sniffing tobacco products, while Co was below the detection limit in the same sample. As and $\mathrm{Cd}$ were below the detection limit in all chewing tobacco brands. Results are summarized in Table 4 and in Figure 1. Codes of all samples have been decoded in Additional file 1: Table S1.

A mean value of all analyzed samples from each category of tobacco products was calculated for various elements and a log-scale comparative graph was drawn
Figure 2. The decreasing order of elemental concentration can be summarized as $\mathrm{Fe}>\mathrm{Al}>\mathrm{Mn}>\mathrm{Zn}>\mathrm{Cu}>$ $\mathrm{Ni}>\mathrm{Cr}>\mathrm{Pb}>\mathrm{Co}>\mathrm{Cd}>\mathrm{As}>\mathrm{Se}$ for smoked tobacco products, $\mathrm{Fe}>\mathrm{Al}>\mathrm{Mn}>\mathrm{Zn}>\mathrm{Cu}>\mathrm{Cr}>\mathrm{Ni}>\mathrm{Pb}>\mathrm{Co}>$ As $>\mathrm{Se}>\mathrm{Cd}$ for sniffing tobacco products, $\mathrm{Fe}>\mathrm{Al}>$ $\mathrm{Mn}>\mathrm{Zn}>\mathrm{Cu}>\mathrm{Cr}>\mathrm{Ni}>\mathrm{Pb}>\mathrm{Co}>\mathrm{As}>\mathrm{Se}>\mathrm{Cd}$ for dipping tobacco products and $\mathrm{Fe}>\mathrm{Al}>\mathrm{Mn}>\mathrm{Zn}>\mathrm{Cu}>$ $\mathrm{Ni}>\mathrm{Cr}>\mathrm{Pb}>\mathrm{Se}>\mathrm{Co}>\mathrm{As}>\mathrm{Cd}$ for chewing tobacco products. Sniffing, dipping and chewing products are different from the smoked products due to their direct contact of tobacco into mucus membrane and metals can be absorbed through mucosal membrane. The recommended dietary intake of metals and metalloids [26-28] is highlighted in the Additional file 1: Table S2. The situation is really alarming as the single dose of these tobacco products is between $2-10 \mathrm{~g}$, while the number

Table 6 Pearson Correlation matrix for 12 elements

\begin{tabular}{|c|c|c|c|c|c|c|c|c|c|c|c|c|}
\hline Variables & Al & $\mathrm{Cr}$ & $M n$ & $\mathrm{Fe}$ & Co & $\mathrm{Ni}$ & $\mathrm{Cu}$ & $\mathrm{Zn}$ & As & $\mathrm{Se}$ & Cd & $\mathrm{Pb}$ \\
\hline$\overline{\mathrm{Al}}$ & 1 & & & & & & & & & & & \\
\hline $\mathrm{Cr}$ & 0.673 & 1 & & & & & & & & & & \\
\hline $\mathrm{Mn}$ & 0.159 & 0.121 & 1 & & & & & & & & & \\
\hline $\mathrm{Fe}$ & 0.962 & 0.683 & 0.146 & 1 & & & & & & & & \\
\hline Co & 0.929 & 0.712 & 0.385 & 0.893 & 1 & & & & & & & \\
\hline $\mathrm{Ni}$ & 0.805 & 0.901 & 0.188 & 0.805 & 0.828 & 1 & & & & & & \\
\hline $\mathrm{Cu}$ & 0.199 & -0.003 & 0.034 & 0.227 & 0.184 & 0.064 & 1 & & & & & \\
\hline $\mathrm{Zn}$ & 0.403 & 0.210 & 0.651 & 0.391 & 0.480 & 0.302 & 0.103 & 1 & & & & \\
\hline As & 0.843 & 0.450 & 0.295 & 0.825 & 0.850 & 0.599 & 0.148 & 0.517 & 1 & & & \\
\hline $\mathrm{Se}$ & 0.315 & 0.100 & 0.272 & 0.235 & 0.317 & 0.127 & -0.157 & 0.293 & 0.546 & 1 & & \\
\hline $\mathrm{Cd}$ & -0.289 & -0.209 & 0.698 & -0.286 & -0.077 & -0.195 & -0.171 & 0.291 & -0.083 & 0.198 & 1 & \\
\hline $\mathrm{Pb}$ & 0.707 & 0.270 & 0.174 & 0.666 & 0.633 & 0.438 & 0.045 & 0.469 & 0.676 & 0.229 & -0.054 & 1 \\
\hline
\end{tabular}

Values in bold show absolute values greater than 0.50 . 
Table 7 Principal component loadings

\begin{tabular}{lcccr}
\hline Variable & PC1 & PC2 & PC3 & PC4 \\
\hline $\mathrm{Al}$ & $\mathbf{0 . 9 5 9}$ & -0.188 & 0.011 & -0.091 \\
\hline $\mathrm{Cr}$ & $\mathbf{0 . 7 2 5}$ & -0.272 & -0.234 & 0.509 \\
\hline $\mathrm{Mn}$ & 0.344 & $\mathbf{0 . 8 2 8}$ & 0.157 & 0.302 \\
\hline $\mathrm{Fe}$ & $\mathbf{0 . 9 4 0}$ & -0.216 & 0.055 & -0.035 \\
\hline $\mathrm{Co}$ & $\mathbf{0 . 9 6 4}$ & 0.006 & 0.024 & 0.084 \\
\hline $\mathrm{Ni}$ & $\mathbf{0 . 8 4 6}$ & -0.227 & -0.139 & 0.396 \\
\hline $\mathrm{Cu}$ & 0.169 & -0.172 & $\mathbf{0 . 8 9 1}$ & -0.061 \\
\hline $\mathrm{Zn}$ & $\mathbf{0 . 5 5 9}$ & $\mathbf{0 . 5 7 7}$ & 0.231 & -0.006 \\
\hline $\mathrm{As}$ & $\mathbf{0 . 8 8 9}$ & 0.103 & -0.018 & -0.322 \\
\hline $\mathrm{Se}$ & 0.381 & 0.415 & -0.409 & -0.495 \\
\hline $\mathrm{Cd}$ & -0.123 & $\mathbf{0 . 8 7 7}$ & -0.026 & 0.237 \\
\hline Pb & $\mathbf{0 . 7 1 6}$ & 0.065 & 0.053 & -0.352 \\
\hline Eigen value & 5.896 & 2.211 & 1.122 & 1.056 \\
\hline Variability (\%) & 49.136 & 18.422 & 9.346 & 8.799 \\
\hline Cumulative Variance (\%) & 49.136 & 67.558 & 76.904 & 85.704 \\
\hline Vales
\end{tabular}

Values in bold show absolute values greater than 0.50 .

of doses consumed per day mainly depends on the level of tobacco addiction of the user, this would be an additional amount of metals taken by the consumer excluding other environmental and nutritional sources.

\section{Comparison with existing data}

Many researchers have quantified the metal contents in smoked tobacco products, particularly in cigarettes [29]. We have compared our data with the reported literature of smoked tobacco products from Pakistan (Table 5). Limited literature is available about sniffing and chewing products and only from India. However, to the best of our knowledge, no report exists about metal contents in dipping tobacco products. In smoked products, our study showed that $\mathrm{Mn}, \mathrm{Cu}$ and $\mathrm{Zn}$ were higher; $\mathrm{Cd}$ has comparable concentration, while $\mathrm{Co}$ and $\mathrm{Pb}$ were lower than the reported concentrations from Pakistan [21]. In the case of sniffing tobacco, most of the metallic contents are nearly comparable with the existing data [30], while $\mathrm{Mn}$ was found to be lower and $\mathrm{Pb}$ as reported by U.C. Mishra et al. [31] was reasonably high among all the reported studies. In case of chewing tobacco, our results showed a little lower concentration of $\mathrm{Cr}, \mathrm{Mn}, \mathrm{Fe}$ and $\mathrm{Pb}$, when compared with existing literature [29-33]. Only one report exists about the quantification of the metalloid, Arsenic, in a chewing tobacco product, but As levels were below the detection limit in our case. The present work is the latest source of information with reference to metals and metalloids contents in tobacco products, especially smokeless tobacco formulations.

\section{Statistical evaluations}

\section{Correlation between variables}

As the principal component analysis is based on Eigen values of correlation matrix, a detailed discussion on correlation matrix is useful in divergence or coherence of data. Data points, whose concentrations were below the detection limit, were replaced by half of detection limit values for the statistical evaluation. Values higher than 0.50 were considered to correlate the data points (Table 6). Positive values in the table show positive correlation among variables while negative values show negative correlation. Values closer to 0 indicate poor negative or positive correlation. However, values nearer to 1 show significant correlation. Neither pairs of elements showed significant negative correlation between them, however, $\mathrm{Cu}$ showed no appreciable correlation to any of the element analyzed. Hence, it was placed in a separate group. The remaining elements were easily grouped in two distinct classes after interpretation of the correlation matrix and are: Group $1=\mathrm{Al}, \mathrm{Fe}, \mathrm{Co}, \mathrm{Ni}, \mathrm{As}$,
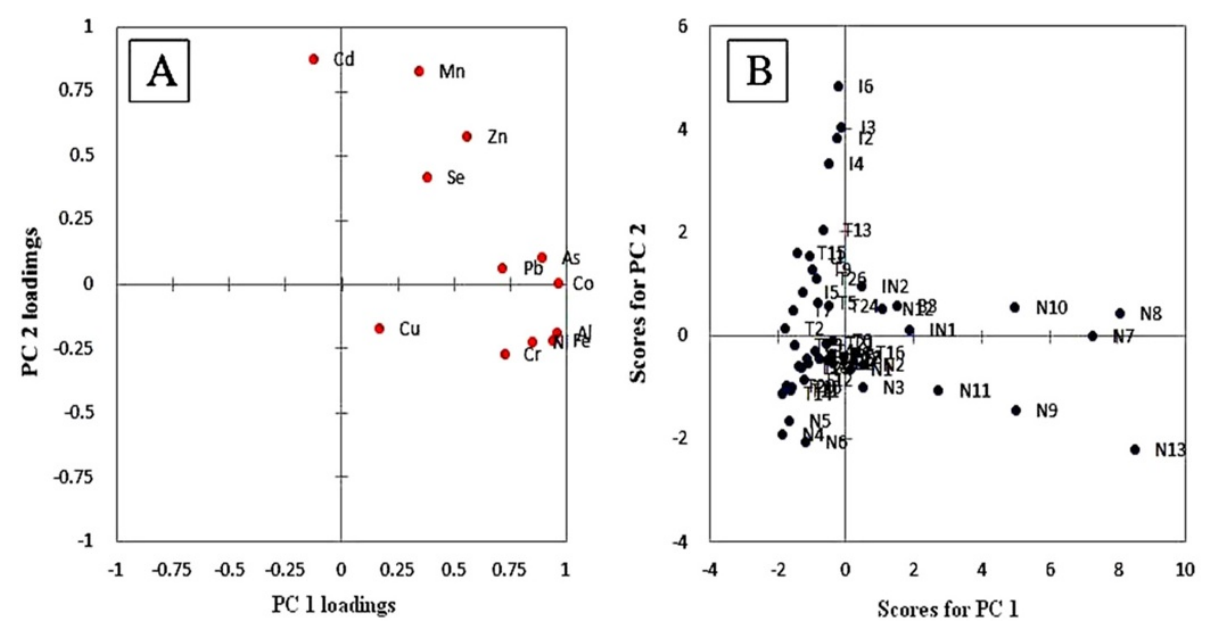

Figure $3 \mathrm{~A}=$ contribution of each element to the $\mathrm{PC}$ loadings and $\mathrm{B}=$ principal component scores for tobacco products. 
Table 8 Factor loadings of Q-mode factor analysis after Varimax rotation

\begin{tabular}{|c|c|c|}
\hline Sample Code & D1 & D2 \\
\hline $\mathrm{T} 1$ & -0.243 & -0.485 \\
\hline $\mathrm{T} 2$ & -0.706 & 0.117 \\
\hline T3 & -0.196 & -0.398 \\
\hline $\mathrm{T} 4$ & -0.473 & -0.186 \\
\hline T5 & -0.636 & 1.003 \\
\hline T6 & -0.349 & 0.086 \\
\hline T7 & -0.593 & 0.397 \\
\hline $\mathrm{T} 8$ & -0.099 & -0.314 \\
\hline T9 & -0.673 & 0.608 \\
\hline T10 & -0.227 & -0.474 \\
\hline T11 & -0.404 & -0.817 \\
\hline T12 & -0.001 & -0.560 \\
\hline T13 & -0.763 & 1.376 \\
\hline T14 & -0.407 & -0.786 \\
\hline T15 & -0.678 & 0.714 \\
\hline T16 & -0.020 & 0.044 \\
\hline $\mathrm{T} 17$ & 0.067 & -0.173 \\
\hline T18 & -0.265 & -0.426 \\
\hline T19 & -0.371 & -0.220 \\
\hline T20 & -0.395 & -0.688 \\
\hline $\mathrm{T} 21$ & -0.361 & -0.122 \\
\hline $\mathrm{T} 22$ & -0.447 & -0.447 \\
\hline $\mathrm{T} 23$ & -0.622 & -0.265 \\
\hline $\mathrm{T} 24$ & -0.566 & 0.525 \\
\hline T25 & -0.334 & -0.604 \\
\hline T26 & -0.566 & 0.333 \\
\hline 11 & -0.640 & 0.928 \\
\hline 12 & -1.212 & 2.712 \\
\hline 13 & -0.814 & 1.646 \\
\hline 14 & -0.829 & 2.243 \\
\hline 15 & -0.697 & 0.756 \\
\hline 16 & -1.050 & 2.562 \\
\hline B1 & -0.159 & -0.571 \\
\hline B2 & 0.242 & -0.506 \\
\hline B3 & 0.419 & 0.520 \\
\hline N1 & 0.115 & -0.562 \\
\hline N2 & 0.524 & -0.673 \\
\hline N3 & 0.069 & -0.574 \\
\hline N4 & -0.166 & -1.469 \\
\hline N5 & -0.302 & -1.410 \\
\hline N6 & 0.094 & -1.535 \\
\hline N7 & 2.844 & 1.279 \\
\hline N8 & 2.920 & 1.304 \\
\hline N9 & 2.404 & -0.501 \\
\hline N10 & 1.626 & 1.280 \\
\hline N11 & 1.073 & 0.088 \\
\hline
\end{tabular}

Table 8 Factor loadings of Q-mode factor analysis after Varimax rotation (Continued)

\begin{tabular}{lrr}
\hline N12 & 0.796 & -0.375 \\
\hline N13 & 3.718 & -0.089 \\
\hline N1 & 0.725 & 0.129 \\
\hline N2 & 0.230 & -0.312 \\
\hline IN3 & 0.676 & 0.488 \\
\hline G2 & -0.871 & -1.385 \\
\hline G3 & -0.085 & -1.791 \\
\hline G4 & -0.531 & -1.496 \\
\hline Eigenvalue & -0.790 & -0.920 \\
\hline Variability (\%) & 5.780 & 2.000 \\
\hline Cumulative Variance (\%) & 44.854 & 19.888 \\
\hline Values in bold show samps $w$ low & 44.854 & 64.742 \\
\hline
\end{tabular}

Values in bold show samples with low loadings in Factor 1 but high loadings in Factor 2.

$\mathrm{Cr}$ and $\mathrm{Pb}$, Group $2=\mathrm{Mn}, \mathrm{Zn}$, Se and $\mathrm{Cd}$, Group $3=\mathrm{Cu}$. Grouping of the data is further elaborated through a multivariate analysis i.e. principal component analysis.

\section{Principal component analysis}

Principal components having Eigen values greater than 1 were extracted for this study. This generated four independent components. The first component contributed as $49.1 \%$, second $18.4 \%$, third $9.3 \%$ and fourth $8.7 \%$ variability of the data. Total contribution from these four components is $85.7 \%$ of the total variation (Table 7 . Analysis of table 7 shows that elements of group 1 gave a major contribution to principal component 1 . The second group of the correlation matrix significantly contributed in factor loadings of principal component 2 . While a unique trend for $\mathrm{Cu}$ was again observed. It showed a significant appearance in principal component 3 and no other element appeared in that factor. This grouping was also clearly apparent in the diagram of loadings of components 1 and 2 (Figure 3A). This figure showed a total of $67.56 \%$ variance of the data, while, Figure 3B showed the score plot for the first two components.

\section{Q-mode factor analysis}

The 55 different tobacco products were subjected to Q-mode factor analysis. This analysis generated many factors, but among them, only two were produced after Varimax rotation. These two factors accounted for $64.7 \%$ of total variability (Table 8). The first factor with $44.8 \%$ variability was considered to be a major factor. This factor may depend on the processing procedure. As a majority of the smoked tobacco products (either local or international) have low loadings in factor 1 and all the smokeless products have high loadings in factor 1 it can be concluded that smokeless tobacco products undergo many processing steps and thus they have more chance 

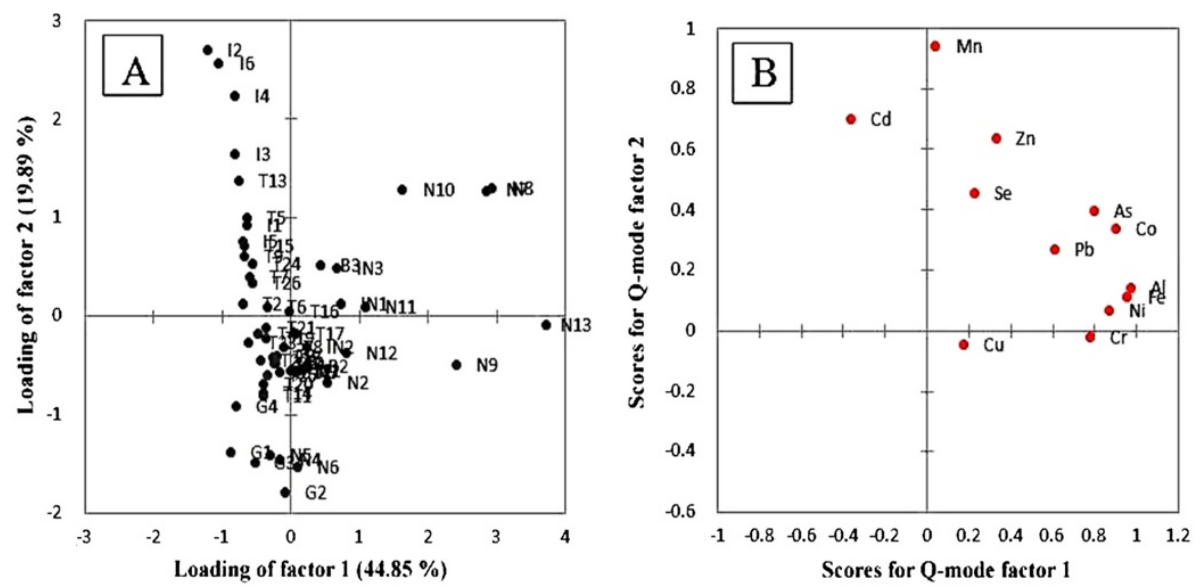

Figure $4 \mathrm{~A}=$ plot of Q-mode factor loadings and $\mathrm{B}=$ plot of Q-mode factor scores.

of elemental contamination. The smoked tobacco products undergo fewer processing steps, hence majority of them gave high loadings in factor 2. Figure $4 \mathrm{~A}$ and $\mathrm{B}$ showed the loadings and scores plot of factors 1 and 2 for the Q-mode factor analysis, respectively.

\section{Conclusions}

This study focused on ICP-MS based quantitative estimation of metals and metalloids in various tobacco products. The method was validated by analyzing certified reference material. Good agreement between recovered and certified values showed effective recovery of the metals after microwave digestion and subsequent accurate detection. The limits of detection for all twelve elements ranged between 0.001-5.23 ng/L, which were much better when compared with the studies reported in literature. The average observed concentration ranges $(\mu \mathrm{g} / \mathrm{g})$ of metals in all types of tobacco products were $\mathrm{Al}$ (111.27-6500), Cr (0.69-78.8), Mn (27.37-300), Fe (176.96-7400), Co (0.06-2.9), Ni (1.19-23.5), Cu (10.537.9), Zn (9.15-66.9), As (0.33-3.07), Se (0.062-2.28), Cd (0.093-3.35) and $\mathrm{Pb}(0.08-4.9)$. The present study provides reliable data about the metals distribution in some commonly consumed tobacco products. Hence, this study would be helpful for toxicologists and environmental chemists to evaluate the health effects of tobacco products and their contribution towards overdosing of the metals in tobacco users.

\section{Additional file}

Additional file 1: Table S1. Decoding of sample codes. Table S2.

Recommended dietary intake of metals and metalloids.

\section{Competing interests}

The authors declare that they have no competing interests.

\section{Acknowledgements}

We gratefully acknowledge the cooperation of Jiri Mizera from "Nuclear Physics Institute of the Academy of Sciences of the Czech Republic (ASCR)" for furnishing us with certified reference material (Oriental tobacco leaves, CTA-OTL-1). This research was made possible, in part, by the RI-INBRE research core facility, supported by grant\# P20RR16457 from NCRR, NIH.

\section{Author details}

'H.E.J. Research Institute of Chemistry, International Center for Chemical and Biological Sciences, University of Karachi, Karachi 75270, Pakistan.

${ }^{2}$ Department of Chemistry, Bahauddin Zakariya University (B.Z.U.), Multan 60800, Pakistan. ${ }^{3}$ College of Pharmacy, University of Rhode Island, Kingston, RI 02881, USA.

\section{Authors' contributions}

SGM: Participated in the experimental designing and method optimization. MS: Participated in bench work and played a role in paper writing. AJS: Involved in statistical evaluations. MNH: Involved in the useful discussion and participated in paper writing. AA: Involved in the useful discussion and participated in performing experiment. All authors read and approved the final manuscript.

Received: 20 January 2012 Accepted: 18 June 2012

Published: 18 June 2012

\section{References}

1. World Health Organization: WHO report on the global tobacco epidemic. Geneva: WHO press; 2008. www.who.int/tobacco/mpower/ mpower_report_full_2008.pdf (Assessed Mar 31, 2009).

2. Hoffmann D, Hoffmann I, El-Bayoumy K: The less harmful cigarette: a controversial issue. A tribute to Ernst L. Wynder. Chem Res Toxicol 2001, 14:767-790.

3. Atrens DM: Nicotine is an addictive substance: a critical examination of the basic concepts and the empirical evidence. J Drug Issues 2001, 32:325-394.

4. Repace JL, Lowrey AH: Indoor air pollution, tobacco smoke and public health. Science 1980, 208:464-472.

5. Wu D, Landsberger S, Larson SM: Determination of the elemental distribution in cigarette components and smoke by instrumental neutron activation analysis. J Radioanal Nucl Chem 1997, 217:77-82.

6. Smith MV, Sneddon J: Simultaneous determination of cadmium and lead in cigarettes and cigarette smoke by impaction-graphite furnace and graphite furnace atomic absorption spectrometry. Spectrosc Lett 1999, 32:615-621.

7. Csalari J, Szantai K: Transfer rate of cadmium, lead, zinc and iron from the tobacco-cut of the most popular Hungarian cigarette brands to the combustion products. Acta Aliment 2002, 31:279-288.

8. Chiba M, Masironi R: Toxic and trace elements in tobacco and tobacco smoke. Bull World Health Org 1992, 70:269-275. 
9. Goyer RA: Toxic effects of metals. In Casarett \& Doull's Toxicology: The Basic Science of Poisons. 5th edition. Edited by Klaasen CD. McGraw-Hill: New York; 1996:691-736.

10. International Agency for Research on Cancer: Tobacco Smoke and Involuntary Smoking, Monographs on the Evaluation of the Carcinogenic Risks of Chemicals to Humans. France 2004, 83:121-844.

11. Trivedy C, Meghji S, Warnakulasuriya KAAS, Johnson NW, Harris M: Copper stimulates human oral fibroblasts in vitro: a role in the pathogenesis of oral sub mucous fibrosis. J Oral Pathol Med 2001, 30:465-470.

12. Neuspiel DR, Markowitz M, Drucker E: Intrauterine cocaine, lead and nicotine exposure and fetal growth. Am J Pub Health 1994, 84:1492-1494.

13. Byrd DM, Roegner ML, Griffiths JC, Lamm SH, Grumski KS, Wilson R, Lai S: Carcinogenic risk of inorganic arsenic in perspective. Int Arch Occup Environ Health 1996, 68:484-494.

14. Jarup $L$, Berglund $M$, Elinder $C H$, Nordberg $G$, Vahter $M$ : Health effects of cadmium exposure and risk estimate. Scand J Work Environ Health 1998, 24:1-52.

15. World Health Organization Regional Office for the Eastern Mediterranean (WHO/EMRO): Tobacco control country profiles 2003. Atlanta GA: American Cancer Society; 2003. http://www.emro.who.int. (Accessed 10 July 2007).

16. Oladipo MOA, Ajayi OO, Elegba SB, Alonge SO, Adeleye SA: The determination of minor and trace elements in some Nigerian cigarettes and raw tobacco using inductively coupled plasma mass spectrometry (ICP-MS). J Environ Sci Health Part A: Environ Sci Eng 1993, 28:839-857.

17. Barlas H, Obay G, Soyhan B, Bayat C: Heavy metal concentrations of cigarettes in Turkey. Fresenius Environ Bull 2001, 10:80-83.

18. Kullab M, Ismail A, Al-Kofahi M: Analysis of Jordanian cigarettes using XRF techniques. Abhath Al-Yarmouk: Pure Sci Eng 2002, 11:87-94.

19. Lugon-Moulin N, Martin F, Krauss MR, Ramey PB, Rossi L: Cadmium concentration in tobacco (Nicotiana tabacum L.) from different countries and its relationship with other elements. Chemosphere 2006, 63:1074-1086

20. Shi J, Li L, Hu QY, Chen ZG, Wang F: Simultaneous determination of chromium, nickel, arsenic, selenium, cadmium, mercury and lead in tobacco with inductively coupled plasma mass spectrometry. Yancao Keji 2006, 37:29-34.

21. Ajab H, Yasmeen S, Yaqub A, Ajab Z, Junaid M, Siddique M, Farooq R, Malik SA: Evaluation of trace metals in tobacco of local and imported cigarette brands used in Pakistan by spectrophotometer through microwave digestion. J Toxicol Sci 2008, 33:415-420.

22. Cai JB, Wang SF, Tang PP, Su QD: Simultaneous determination of total nitrogen and metal elements in tobaccos by high performance ion chromatography. J Chin Chem Soc 2009, 56:671-675.

23. Dhaware D, Deshpande A, Khandekar RN, Chowgule R: Determination of toxic metals in Indian smokeless tobacco products. The ScientificWorld 2009, 9:1140-1147.

24. Kazi TG, Jalbani N, Arain MB, Jamali MK, Afridi HI, Sarfraz RA, Shah AQ: Toxic metals distribution in different components of Pakistani and imported cigarettes by electrothermal atomic absorption spectrometer. 1 Hazard Mater 2009, 163:302-307.

25. Swami K, Judd CD, Orsini J: Trace metals analysis of legal and counterfeit cigarette tobacco samples using inductively coupled plasma mass spectrometry and cold vapor atomic absorption spectrometry. Spectrosc Lett 2009, 42:479-490.

26. N.S.F. International: Dietary Supplement-Standard 173, Metal Contaminant Acceptance levels. USA: USA; 2003. www.nsf.org/business/newsroom/pdf/ DS_Metal_Contaminant_Acceptance_Levels.pdf.

27. Raghunath $\mathrm{R}$, Tripathi RM, Suseela B, Bhalke S, Shukla VK, Puranik VD: Dietary intake of metals by Mumbai adult population. Sci Total Environ 2006, 356:62-68

28. Nordberg GF, Fowler BA, Nordberg M, Friberg M: Handbook on the toxicology of metals. 3rd edition. London: Academic; 2007.

29. Verma S, Yadav S, Singh I: Trace metal concentration in different Indian tobacco products and related health implications. Food Chem Toxicol 2010, 48:2291-2297.

30. Shaikh AN, Negi BS, Sadasivan S: Characterization of Indian cigarette tobacco and its smoke aerosol by nuclear and allied techniques. J Radioanal Nucl Chem 2002, 253:231-234.

31. Mishra UC, Shaikh GN, Sadasivan S: Trace elements in tobacco and tobacco smoke by X-Ray fluorescence technique. J Radioanal Nucl Chem 1986, 102:27-35.
32. Mishra UC, Shaikh GN: Simultaneous multielement determination of chewing and snuff tobaccos used in India by INNA. J Radioanal NuC Chem 1986, 98:297-301.

33. Mishra UC, Shaikh GN: Determination of trace element concentrations of Indian cigarette tobacco by instrumental neutron activation analysis. J Radioanal Chem 1983, 78:385-390.

doi:10.1186/1752-153X-6-56

Cite this article as: Musharraf et al:: Quantitative analysis of some important metals and metalloids in tobacco products by inductively coupled plasma-mass spectrometry (ICP-MS). Chemistry Central Journal 2012 6:56.

\section{Publish with ChemistryCentral and every scientist can read your work free of charge \\ "Open access provides opportunities to our colleagues in other parts of the globe, by allowing anyone to view the content free of charge." W. Jeffery Hurst, The Hershey Company. \\ - available free of charge to the entire scientific community \\ - peer reviewed and published immediately upon acceptance \\ - cited in PubMed and archived on PubMed Central \\ - yours - you keep the copyright \\ Submit your manuscript here: \\ http://www.chemistrycentral.com/manuscript/<smiles>c1ccccc1</smiles> \\ Chemistry Central}

